\title{
Controlled Fusion Strategy Using Ultra-Intense Laser Derived Positron Generation for Initiation
}

\author{
Robert Le Moyne \\ Independent (Senior Member AIAA and Senior Member IEEE) Running Springs, CA, USA \\ Email: rlemoyne07@gmail.com
}

How to cite this paper: Le Moyne, R. (2018) Controlled Fusion Strategy Using Ultra-Intense Laser Derived Positron Generation for Initiation. Journal of Applied Mathematics and Physics, 6, 693-703. https://doi.org/10.4236/jamp.2018.64062

Received: March 19, 2018

Accepted: April 9, 2018

Published: April 12, 2018

\begin{abstract}
A controllable strategy for eliciting nuclear fusion is presented through ultra-intenselaser derived positron generation by a conceptual first physics perspective. The capability to generate positrons on demand in a controlled manner through an ultra-intense laser incident on a high atomic number target, such as gold, is the intrinsic core to the foundation of controllable nuclear fusion. Positron antimatter generated from the periphery of the fusion fuel pellet provides the basis for initiating the fusion reaction, which is regulated by controlling the operation of the ultra-intense laser. A dual pulsed Fast Ignition mechanism is selected to achieve the fusion reaction. Based on first physics performance analysis the controllable strategy for eliciting nuclear fusion through ultra-intenselaser derived positron generation offers a realizable means for achieving regulated nuclear fusion. A future perspective of the controllable fusion strategy addresses the opportunities and concerns of a pathway toward regulated nuclear fusion.
\end{abstract}

\section{Keywords}

Controllable Nuclear Fusion, Ultra-Intense Laser, Positron, Positron

Generation, Antimatter, Trident Process, Bethe-Heitler Process,

Breit-Wheeler Process, Volumetric Ignition, Hotspot Ignition, Fast Ignition

\section{Introduction}

The advent of the ultra-intense laser has commenced the capacity to generate positron antimatter [1] [2]. The capacity to generate positron antimatter through the incidence of ultra-intense lasers has enabled new perspectives for non-chemical space propulsion. These antimatter derived propulsion systems 
apply the generation of positron antimatter from an ultra-intense laser incident on a high atomic number target, such as gold. One of the advantages of this configuration is the energy source for the laser system can be remotely situated from the propulsion system [3]-[8]. In particular, LeMoyne established a first-physics performance analysis of a space propulsion system utilizing fusion initiated by positron antimatter generated by an ultra-intense laser. Due to the nature of the ultra-intense laser generation of the positron antimatter the mechanisms for fusion can be controlled through the operation of the ultra-intense laser source [8].

Simple nuclear fusion reactions involve the fusion between two hydrogen nuclei, such as deuterium and tritium, that yields helium and a neutron with the release of a considerable quantity of energy as described in Equation (1) [9].

$$
\text { Fusion : }{ }_{1}^{2} \mathrm{H}+{ }_{1}^{3} \mathrm{H} \rightarrow{ }_{2}^{4} \mathrm{He}+{ }_{0}^{1} \mathrm{n} \quad \text { [9] }
$$

The current strategy for achieving nuclear fusion is through the initiation of a nuclear fission event. Although this strategy releases a considerable amount energy, this approach is not a controllable means of eliciting fusion [10]. By contrast the mechanisms for evoking nuclear fusion by LeMoyne constitute a controllable means of eliciting a nuclear fusion event, which has been elucidated from a first physics perspective regarding fundamental performance analysis for the domain of space propulsion [8].

Essential to evoking a controllable means of fusion is the generation of antimatter [8]. Antimatter offers an extremely dense means of storing energy [11]. There are two forms of antimatter: antiproton and positron antimatter. The antiproton represents a greater energy content, since it is the mass equivalent of the proton. By contrast the positron is the antimatter cognate of the electron, which has a lower mass energy content relative to the antiproton and proton [9].

However, the antiproton requires complex storage techniques prior to application for the initiation of fusion. Positrons can be generated on demand through the incidence of an ultra-intense laser on a high atomic number target, such as gold [3]-[8]. There are three mechanisms for generating antimatter through the incorporation of an ultra-intense laser: Trident process, Bethe-Heitler process, and Breit-Wheel erprocess [12] [13] [14]. Each of these mechanisms have unique characteristics for their suitable application. Furthermore, there are three mechanisms for eliciting a fusion reaction by antimatter initiation: Volumetric Ignition, Hotspot Ignition, and Fast Ignition [15].

The objective is to elucidate from a first-physics perspective performance analysis of fusion initiated by positron generation through an ultra-intense laser on a high atomic number target, such as gold. Given the advantages, the Bethe-Heitler mechanism is applied for the generation of positron antimatter through an ultra-intense laser incident on a high atomic number target, such as gold, surrounding the periphery of the fusion fuel pellet. Given the benefits of the energy requirement for commencing fusion and yield, the Fast Ignition mechanism is applied to establish a means of controllable fusion. Furthermore, some of the considerable opportunities associated with controllable nuclear fu- 
sion are addressed.

\section{Background}

\subsection{Mechanisms for Generating Positrons Derived by Ultra-Intense Laser}

The theoretical basis for imparting laser energy for the generation of positrons was developed by Shearer et al. during the early aspect of the 1970's. In order to achieve the positron generation through laser incidence, lasers achieving the intensity threshold of $10^{20} \mathrm{~W} / \mathrm{cm}^{2}$ were required [16]. Further evolution of laser technology was necessary to achieve this threshold parameter.

Three mechanisms for the generation of positron antimatter through ultra-intense laser interaction are the Trident process, Bethe-Heitler process, and Breit-Wheeler process [12] [13] [14]. The Trident process and Bethe-Heitler process involve interaction with the electron cloud and associated nuclei of a preferably high atomic number target, such as gold [1] [14]. By contrast the Breit-Wheeler process generates positrons through photonic interaction [12] [13].

The Trident process and Bethe-Heitler process generate positron antimatter through ultra-intense laser incidence on a target with preferably high atomic number target, such as gold. The Trident process requires a single step for generating positrons, and this mechanism is most prevalent for targets, such as gold, on the order of 3.5 micrometers thick [1] [14]. The Trident process is summarized through Equation (2):

$$
\mathrm{e}^{-}+\mathrm{Z} \rightarrow \mathrm{e}^{+}+2 \mathrm{e}^{-}+\mathrm{Z}
$$

$$
\begin{aligned}
& \mathrm{e}^{+} \text {: Positron; } \\
& \mathrm{e}^{-} \text {: Electron; } \\
& \text { Z: High atomic number nuclei(such as gold) [1] [14]. }
\end{aligned}
$$

By contrast the Bethe-Heitler process incorporates a dual step mechanism for generating positron antimatter. First the resulting fast electrons produce high-energy bremsstrahlungphotons. Thebremsstrahlungphotons interact with the nuclei, producing positron and electron pairs [1] [14]. The Bethe-Heitler process is detailed in Equations (3) and (4):

$$
\begin{array}{r}
\mathrm{e}^{-}+\mathrm{Z} \rightarrow \gamma+\mathrm{e}^{-}+\mathrm{Z} \\
\gamma+\mathrm{Z} \rightarrow \mathrm{e}^{+}+\mathrm{e}^{-}+\mathrm{Z}
\end{array}
$$

$\gamma$. Bremsstrahlung photons [1] [14].

The Bethe-Heitler process pertains to targets, such as gold, with thickness on the order of $1 \mathrm{~mm}$. Furthermore, the cross-section of the Bethe-Heitler process is on the order of 100 times greater than the Trident process [14].

The third process is the Breit-Wheeler process. The uniqueness of this process is the observation that it only requires photonic interaction [12] [13]. The Breit-Wheeler process is summarized through the simple method summarized in Equation (5): 


$$
\gamma+\gamma \rightarrow \mathrm{e}^{+}+\mathrm{e}^{-}[12][13]
$$

Lawrence Livermore National Laboratory successfully applied their Titan laser for the generation of positron antimatter. The Titan laser realized the $10^{20} \mathrm{~W} / \mathrm{cm}^{2}$ ultra-intense scale proposed by Shearer for positron antimatter generation. The predominant mechanism was the Bethe-Heitler process [1] [2].

The performance attributes of the Titan laser experiment feature the generation of $2 \times 10^{10}$ positrons. The laser pulse of approximately 1 ps was incident on a $1 \mathrm{~mm}$ target composed of gold. Roughly $90 \%$ of the positrons were discharged anisotropic and aft relative to the incidence of the ultra-intense laser beam on the target, which implicates $1.8 \times 10^{10}$ positrons per 1 ps pulse in the aft direction of the target interaction. The positron kinetic energy is on the order of 2 $\mathrm{MeV}$ [1] [2].

Further experimentation of the use of ultra-intense lasers for generating positron antimatter has revealed nuclei with atomic numbers lower than gold are feasible. Simulations have established that nuclei's spanning from lithium $(z=3)$ to uranium $(z=92)$ are capable of generating positrons through ultra-intense laser interaction while emphasizing the Bethe-Heitler process. The trend corresponds to a diminishing quantity of positrons with a decrease in atomic number [17].

\subsection{Antimatter Mechanisms for Eliciting Nuclear Fusion}

There are three mechanisms to be considered for the objective of positron derived controllable nuclear fusion. These mechanisms are derived from the origins of antiproton antimatter catalyzed nuclear fusion [15]. As opposed to the application of a stored and then directed source of antiproton antimatter, the use of positrons derived by ultra-intense laser pulses on a target, such as gold, provides the energy source for commencing the nuclear fusion event. These three mechanisms under consideration are:

- Volumetric Ignition;

- Hotspot Ignition;

- Fast Ignition [15].

\subsubsection{Volumetric Ignition}

The Volumetric Ignitionmechanism involves the pre-compression phase of a deuterium and tritium fuel with a heavy metal seed, such as uranium. The beam of antiproton antimatter is injected in to the pre-compressed fusion fuel with seeding. Pre-compression is achieved by conventional or antiproton ablative drive. A unique feature of this mechanism is the super fission of uranium as a consequence of interaction with antiprotons [15].

The nuclear yield is a consequence of antimatter annihilation from incident antiprotons, nuclear fusion from deuterium and tritium, and nuclear fission based on seed uranium. Antiproton requirements are on the order of $6.5 \times 10^{17}$. Then energy yield is estimated on the order of $404 \mathrm{MJ}$ with a seed fraction on the order of $5 \%$ [15]. 
However, the Volumetric Ignition mechanism conflicts with the objective of utilizing positrons derived from the incidence of an ultra-intense laser as alternative to antiprotons. Uranium interacting with antiprotons augments the fusion yield with super fission. However, the capacity of positron interaction with heavy metal is an undefined mechanism. Therefore, the antiproton Volumetric Ignitionmechanism is precluded from further consideration.

\subsubsection{Hotspot Ignition}

The Hotspot Ignition mechanism has been previously addressed from a first physics conceptual perspective as a controlled form of ultra-intense laser derived position initiated fusion propulsion. This form of space propulsion enables the decoupling of the laser energy source and fusion propulsion system. This propulsion configuration offers advantageous performance capabilities with a considerable specific impulse [8].

The mechanism for the Hotspot Ignition consists of a heavy metal seeded ablator about the surrounding peripheral. Central to the pellet core is the deuterium and tritium nuclear fusion fuel. This strategy inherently enables essentially clean fusion reaction. The antiproton Hotspot Ignition mechanism offers higher gains, as a relatively smaller quantity of the fusion fuel is fuel is required to achieve the ignition temperature threshold. The implication of this configuration is the reduction of antiprotons incident on the pellet target [15].

The quantity of antiprotons incident on the Hotspot Ignition mechanism fusion target are approximately $1.9 \times 10^{16}$, which is effectively an order of magnitude less the Volumetric Ignition methodology. The heavy metal seed for the intent of the ablator is scaled to $20 \%$. The yield associated with the antiproton injection is on the order of $560 \mathrm{MJ}$ [15]. The utility and performance capabilities of the antiproton induced Hotspot Ignition mechanism warrants further consideration as a viable form of regulated and controlled nuclear fusion through the incidence of ultra-intense laser derived positrons.

\subsubsection{Fast Ignition}

The Fast Ignition mechanism applies antiprotons to initiate fusion in a manner that conceptually resembles the Hotspot Ignition mechanism. The major disparity between the two mechanisms is that the Fast Ignition mechanism achieves nuclear fusion through two segmented pulses of antimatter on different time scales. In essence, the compression and ignition phases are decoupled [15].

The preliminary pulse of antimatter is applied to compress the fusion fuel source to an appropriate density. The associated time scale is on the order of 100 nanoseconds. The secondary pulse provides the fusion fuel ignition. The time scale for the ignition pulse of antimatter is relatively faster with a time scale of approximately 10 picoseconds. Because this strategy involves reduction in preliminary compression energy requirements the yield gains of the fusion fuel are anticipated to be on a greater scale relative to the Hotspot Ignitionmechanism [15].

The antiproton requirement for the Fast Ignition mechanism is appropriately 
an order of magnitude less than the requirements featured for the Hotspot Ignition mechanism. The preliminary compression pulse of antiproton antimatter is approximately $2.5 \times 10^{15}$ antiprotons, and the pending ignition pulse applies 0.62 $\times 10^{15}$ antiprotons. The Fast Ignition mechanism fusion target would resemble the Hotspot Ignition fusion target. The inner layer would consist of a deuterium-tritium core that would be encased by an outer layer seeded with ablator in a manner resembling the Hotspot Ignition mechanism configuration. As the fusion target configuration of the Fast Ignition mechanism is similar to the Hotspot Ignition mechanism the associated yield using the injection of antiproton antimatter is on the order of $560 \mathrm{MJ}$. However, since the Fast Ignition mechanism applies a dual stage compression then ignition approach, which reduces the antimatter requirement for achieving fusion, the gain is amplified on the order of six-fold [15]. Given the implied opportunity and performance characteristics of the Fast Ignition mechanism, further consideration is warranted as a viable configuration for regulated and controlled nuclear fusion based on ultra-intense laser derived positrons.

\section{First Physics Fundamental Performance Analysis of Fusion Initiated by Positrons Derived from Ultra-Intense Laser}

Controllable nuclear fusion can be elicited through the Hotspot Ignition and Fast Ignition mechanisms. As an alternative to antiproton antimatter, for which storage issues exist, positrons are generated through an ultra-intense laser incident on a high atomic number target such as gold. The advantage of initiating these fusion mechanisms through positrons generated by ultra-intense laser pulses is the controllable nature of nuclear fusion through this strategy. Furthermore, the laser energy source can be remotely situated from the fusion event.

Further analysis requires the energy definition of the positron and quantified contrast to the antiproton. From a rest mass perspective, the positron and electron pair the energy is on the order of $2 \mathrm{mc}^{2}=1.02 \mathrm{MeV}$. Kinetic energy is conserved and assumed elastic [9] [18]. Given, the $2 \mathrm{MeV}$ kinetic energy of the positron, the positron-electron annihilation event is estimated to be $3.02 \mathrm{MeV}$ [4]. The rest mass energy of a proton and associated antiproton is roughly $938 \mathrm{MeV}$; therefore, the proton-antiproton annihilation event would represent energy of approximately $1876 \mathrm{MeV}$ [9]. Therefore, approximately 621 positrons would constitute the equivalent energy yield of an antiproton annihilation event [8]. With this positron to antiproton energy ratio established, further first physics fundamental performance analysis regarding the Hotspot Ignition and Fast Ignition mechanisms for nuclear fusion from positrons generated by ultra-intense laser pulses are investigated.

\subsection{Hotspot Ignition}

The deuterium-tritium fusion fuel would consist of a core mass of $5 \mathrm{mg}$. The 
yield corresponds to $560 \mathrm{MJ}$ [15]. A total of $1.2 \times 10^{19}$ positrons generated per gold target through the application of ultra-intense laser pulses would commence fusion through the Hotspot Ignition mechanism [8]. The fusion pellet would consist of deuterium-tritium core with a concentric peripheral layer comprised of deuterium-tritium integrated with a uranium heavy metal seed. The pellet would be further encompassed with gold to enable the generation of positron antimatter through an ultra-intense laser system. Based on this ultra-intense laser positron antimatter generation configuration approximately 6.7 $\times 10^{8}$ laser pulses would be required to elicit the Hotspot Ignition strategy for nuclear fusion [8].

\subsection{Fast Ignition}

The Fast Ignition mechanism corresponds to an equivalent deuterium-tritium fusionfuel core mass of $5 \mathrm{mg}$. The Fast Ignition mechanism requires approximately 6 times less antiprotons, due to its characteristic nature of segmented compression events. A total of $\sim 3.12 \times 10^{15}$ antiprotons are injected in a segmented manner toward the Fast Ignition fusion pellet target. The yield likewise corresponds to $560 \mathrm{MJ}$ [15]. A total of $1.9 \times 10^{18}$ positrons generated by pulses an ultra-intense laser incident on a concentric periphery of gold surrounding the deuterium-tritium fusionfuelcore pellet would initiate the Fast Ignition mechanism. This ultra-intense laser positron antimatter generation configuration using the Fast Ignition mechanism to achieve nuclear fusion would require $1.1 \times 10^{8}$ laser pulses.

In essence, the Fast Ignition mechanism would correspond to a reduction in laser pulses by a factor of six when contrasted to the Hotspot Ignition mechanism. Therefore, with respect to the three mechanisms to initiate nuclear fusion through antimatter, the Fast Ignition mechanism offers the most superior performance characteristics from a first physics perspective. Furthermore, the generation of positron antimatter from an ultra-intense laser with incident pulses on a gold target constitutes a means of controlling nuclear fusion through operational control of the ultra-intense laser.

The application of positrons derived from an ultra-intense laser for initiating fusion through the Fast Ignition mechanism offers a controllable strategy for nuclear fusion as an energy source. The positron antimatter is generated on demand through an ultra-intense laser targeting a high atomic number substrate, such as gold, that encompasses the Fast Ignition designed fusion pellet. Therefore, the initiation of fusion is regulated through the operation of the ultra-intense laser for generating positrons. Another advantage of the concept is the release of fusion energy may be progressively scaled according to requirement constraints.

\section{Future Concepts and Perspectives}

With the performance characteristics elucidated from a first physics perspective 
future concepts and perspectives of this Fast Ignition mechanism initiated through positrons generated by ultra-intense lasers are considered.

\subsection{Controllable Nuclear Fusion}

As the proposed ultra-intense laser derived positron initiated fusion strategy through the Fast Ignition mechanism is regulated by laser activation and essentially scale able in nature, other scientific and engineering developments may further advance the economic viability of this methodology of controlled fusion. For example, integrating lithium into the fusion fuel pellet may augment the yield, since lithium can undergo fission while yielding products amenable to fusion [10]. Furthermore, the ultra-intense laser generation of positrons is suitable for nuclei spanning from lithium to uranium [17]. This observation enables the consideration of more economically feasible target alternatives relative to gold encompassing the Fast Ignition fusion fuel pellet. Also, uranium could consolidate the function of high atomic number positron generating target while further providing ablator properties. Three-dimensional printing of the fusion fuel pellet could provide optimal quality control of yield, which would be especially valuable for commercial applications. In the event that the timing sequence of the Fast Ignition mechanism for dual dosages of positron antimatter is a matter of challenge, the Breit-Wheeler mechanism for photonically generating positron antimatter may be considered to better realize the Fast Ignition mechanism for controllable nuclear fusion.

\subsection{Space Applications: Near/Deep Space and Missile Defense}

The proposed ultra-intense laser derived positron initiated fusion strategy through the Fast Ignition mechanism can be applied to facilitate both near and potentially deep space missions with regards to their energy requirements. As noted the ultra-intense laser with energy source and fusion pellet target can be remotely separated. For example, the ultra-intense laser platform could be situated in low Earth orbit with energy derived from an array of solar cells. The gold encompassed Fast Ignition designed fusion pellet target could be situated remotely on the Moon (near space) or Mars (relatively deep space). In essence, controlled nuclear fusion could be generated on demand through remote operation of the ultra-intense laser platform, which could greatly expand space mission capabilities.

By turning the proposed ultra-intense laser derived positron initiated fusion strategy through the Fast Ignition mechanism in an Earthbound direction a robust missile defense system could be developed. The ultra-intense laser platform with solar energy source could be aimed Earthbound. With the proper discharge of fusion fuel pellets aimed toward a missile trajectory while scaled in a manner to mitigate collateral damage, the triggering of a controlled and on-demand nuclear fusion detonation could generate a sufficient electromagnetic pulse to considerably disrupt and disable the missile electronic equipment, such as guid- 
ance, navigation, and control. Such a system may constitute a viable missile defense system.

\subsection{Fourth Generation Nuclear Bomb and Counter-Terrorism Concern}

Although the first physics performance analysis of the ultra-intense laser derived positron initiated fusion strategy offers considerable opportunity for the engineering and scientific community, a sobering realization is that the ultra-intense laser derived positron initiated fusion strategy can be applied to the development of a fourth-generation nuclear bomb. Dr. Gsponer of the Independent Scientific Research Institute (ISRI) defines a fourth-generation nuclear bomb as follows:

"Nuclear explosive devices based on low-yield thermonuclear pellets triggered by compact non-fission primaries." [19]

The issue of concern is that a fourth-generation nuclear bomb can be openly research, developed, tested, and evaluated in compliance with the Comprehensive Test Ban Treaty (CTBT) [20]. Since the ultra-intense laser derived positron initiated fusion strategy is envision as a peaceful means for developing controllable nuclear fusion, the author would respectfully encourage the development of proactive international policy to mitigate adverse weapons proliferation of this fusion strategy.

Trends regarding the miniaturization are evident as ultra-intense lasers are capable of being developed in tabletop configurations [21]. Envisioned trends pertain to further advances in miniaturization and also evolution toward greater energy storage systems for activating laser systems. Recently, magneto-hydrodynamics were considered as an electrical energy source for an ultra-intense laser using the exhaust from a rocket nozzle while in flight [22].

A counter-terrorism concern is also apparent, especially when considering the evolution of laser technology over the course of the next two decades. The operational capabilities of a tabletop laser have been realized in an academic environment [21]. Furthermore, positrons can be generated between the span of lithium to uranium. If an academic resource can assemble an ultra-intense laser consolidated to a tabletop configuration, then nuclear terrorists may also be able to emulate this capability. The prospective of a nuclear terrorist attack unleashing a nuclear fusion detonation presents challenge to the counter-intelligence community for the security of all countries.

The author presents the following counter-terrorism perspective to the counter-intelligence community. The bill of materials is presumed to be highly unique for the assembly of a tabletop ultra-intense laser. Therefore, counter-intelligence assets could apply machine learning pattern recognition techniques for the identification of people of interest seeking to purchase the component level and system level aspects of a tabletop configuration ultra-intense laser, enabling proactive intervention. 


\section{Conclusion}

A controllable strategy for eliciting nuclear fusion has been presented from a conceptual first physics perspective. An inherent aspect of the strategy is the ability to generate positron antimatter in a controlled and on-demand manner through an ultra-intense laser incident on a high atomic number target, such as gold. The gold surrounding the periphery of a Fast Ignition designed fusion fuel pellet generates positron antimatter in a regulated manner through the incidence of an ultra-intense laser. The positron antimatter occurs in two pulses sufficient to satisfy the antimatter requirements of the Fast Ignition mechanism for nuclear fusion. On the order of $1.9 \times 10^{18}$ positrons are generated through the Bethe-Heitler process from an ultra-intense laser through $1.1 \times 10^{8}$ laser pulses. The proposed fusion pellet configuration yields $560 \mathrm{MJ}$ of energy through the Fast Ignition mechanism, which has a gain of six times greater than the Hotspot Ignition mechanism. A future perspective is provided on the advancement of this controllable fusion strategy, such as alternative lower atomic number targets, three-dimensional printing of fusion pellets, and the Breit-Wheeler process as an alternative positron generation approach. Alternative applications are discussed, such as an energy source for space missions and missile defense scenarios. Since the controlled fusion strategy using ultra-intense laser derived positron generation for initiation is not regulated by the Comprehensive Test Ban Treaty, concerns of the controlled fusion strategy being applied as a fourth-generation nuclear bomb are addressed. As the ultra-intense laser can be assembled in a tabletop configuration using academic resources, the threat of nuclear terrorism is discussed with a mitigating nuclear counterterrorism advisement provided.

\section{References}

[1] Chen, H., Wilks, S.C., Bonlie, J.D., Chen, S.N., Cone, K.V., Elberson, L.N., Gregori, G., Meyerhofer, D.D., Myatt, J., Price, D.F., Schneider, M.B., Shepherd, R., Stafford, D.C., Tommasini, R., Van Maren, R. and Beiersdorfer, P. (2009) Making Relativistic Positrons Using Ultraintense Short Pulse Lasers. Physics of Plasmas, 16. https://doi.org/10.1063/1.3271355

[2] Chen, H., Wilks, S.C., Bonlie, J.D., Liang, E.P., Myatt, J., Price, D.F., Meyerhofer, D.D. and Beiersdorfer, P. (2009) Relativistic Positron Creation Using Ultraintense Short Pulse Lasers. Physical Review Letters, 102. https://doi.org/10.1103/PhysRevLett.102.105001

[3] LeMoyne, R. (2012) Fundamental Architecture and Analysis of Ramjet Propulsion System Utilizing in-Situ Laser Derived Antimatter. Proceedings of 18 th AIAA/3 AF International Space Planes and Hypersonic Systems and Technologies Conference, Tours, September. https://doi.org/10.2514/6.2012-5863

[4] LeMoyne, R. and Mastroianni, T. (2014) Fundamental Architecture and Analysis of an Antimatter Ultra-Intense Laser Derived Pulsed Space Propulsion System. Journal of Applied Mathematics and Physics, 2, 10-18. https://doi.org/10.4236/jamp.2014.25002

[5] LeMoyne, R. and Mastroianni, T. (2014) Advanced Concept Ramjet Propulsion System Utilizing in-Situ Positron Antimatter Derived from Ultra-Intense Laser with Fundamental Performance Analysis. Journal of Applied Mathematics and Physics, 


\section{2, 19-26. https://doi.org/10.4236/jamp.2014.25003}

[6] LeMoyne, R. and Mastroianni, T. (2014) Fundamental Architecture and Analysis of an Antimatter Ultra-Intense Laser Derived Combined Cycle Ramjet-Rocket Propulsion System. Proceedings of 19 th AIAA International Space Planes and Hypersonic Systems and Technologies Conference, Atlanta, June.

[7] LeMoyne, R. and Mastroianni, T. (2015) Fundamental Architecture and Performance Analysis of Photofission Pulsed Space Propulsion System Using Ultra-Intense Laser. Journal of Applied Mathematics and Physics, 3, 436-442. https://doi.org/10.4236/jamp.2015.34055

[8] LeMoyne, R. (2017) Positron Induced Fusion Pulsed Space Propulsion through an Ultra-Intense Laser. Journal of Applied Mathematics and Physics, 5, 813-821. https://doi.org/10.4236/jamp.2017.54070

[9] Serway, R.A., Moses, C.J. and Moyer, C.A. (1989) Modern Physics. Saunders College, Philadelphia.

[10] Loeber, C.R. (2005) Building the Bombs: A History of the Nuclear Weapons Complex. Sandia National Laboratories, Albuquerque.

[11] Harris, D., Bille, M. and Reed, L. (2003) Future Space Transportation Technology: Prospects and Priorities. Proceedings of Space 2003, Long Beach, September.

[12] Breit, G. and Wheeler, J.A. (1934) Collision of Two Light Quanta. Physical Review, 46. https://doi.org/10.1103/PhysRev.46.1087

[13] Zhu, X.L., Yu, T.P., Sheng, Z.M., Yin, Y., Turcu, I.C. and Pukhov, A. (2016) Dense GeV Electron-Positron Pairs Generated by Lasers in Near-Critical-Density Plasmas. Nature Communications, 7. https://doi.org/10.1038/ncomms13686

[14] Nakashima, K. and Takabe, H. (2002) Numerical Study of Pair Creation by Ultraintense Lasers. Physics of Plasmas, 9, 1505-1512. https://doi.org/10.1063/1.1464145

[15] Perkins, L.J., Orth, C.D. and Tabak, M. (2003) On the Utility of Antiprotons as Drivers for Inertial Confinement Fusion, Lawrence Livermore National Laboratory. Department of Energy, UCRL-TR-200850.

[16] Shearer, J.W., Garrison, J., Wong, J. and Swain, J.E. (1973) Pair Production by Relativistic Electrons from an Intense Laser Focus. Physical Review A, 8, 1582-1588. https://doi.org/10.1103/PhysRevA.8.1582

[17] Williams, G.J. (2016) Studies of Positron Generation from Ultraintense Laser-Matter Interactions, Lawrence Livermore National Laboratory. Department of Energy, LLNL-TH-691086.

[18] Serway, R.A. (1990) Physics for Scientists and Engineers. Saunders College, Philadelphia.

[19] Gsponer, A. (2008) Fourth Generation Nuclear Weapons: Military Effectiveness and Collateral Effects. Independent Scientific Research Institute, ISRI-05-03.17.

[20] Gsponer, A. (2008) From the Lab to the Battlefield? Nanotechnology and Fourth Generation Nuclear Weapons. Independent Scientific Research Institute, ISRI-02-06.10.

[21] Schwoerer, H., Ewald, F., Sauerbrey, R., Galy, J., Magill, J., Rondinella, V., Schenkel, R. and Butz, T. (2003) Fission of Actinides Using a Tabletop Laser. EPL (Europhysics Letters), 61, 47-52. https://doi.org/10.1209/epl/i2003-00243-1

[22] LeMoyne, R. and Mastroianni, T. (2016) Project New Orion: Pulsed Nuclear Space Propulsion Using Photofission Activated by Ultra-Intense Laser. Journal of Applied Mathematics and Physics, 4, 630-636. https://doi.org/10.4236/jamp.2016.44071 